\title{
Formación en valores desde el Área de Psicología del Deporte del Sevilla F.C. S.A.D.
}

\author{
José Ignacio Navarro Díaz, Juan Manuel Gamito Manzano, Mercedes Valiente Martín, José Alejandro Domínguez \\ Catalán y Alejandro Correa Palacios \\ Área de Psicología del Sevilla F.C. S.A.D., España
}

RESUMEN: Una de las máximas motivaciones del Sevilla F.C es educar en valores y formar a sus jugadores, marcándose como tarea fundamental dentro del trabajo que realiza el Área de Psicología del Deporte con los escalafones inferiores del club. La educación en valores busca que los jugadores adquieran comportamientos, conocimientos y actitudes adecuadas para la vida y el deporte, como es el respeto, la tolerancia y el fair-play. Los objetivos del presente trabajo son exponer algunos de los pilares y proyectos del Área de Psicología del Deporte del Sevilla F.C en relación a la educación y formación integral de sus jugadores, destacando la escuelas en valores para las familias, la gestión de los jugadores de la residencia, el apoyo psicopedagógico para la cantera y el proyecto escolar "Sácale Partido al Cole". Se concluye que el asesoramiento a padres, el entrenamiento en valores y los estudios contribuye al crecimiento personal y desarrollo deportivo de los jóvenes jugadores.

PALABRAS CLAVES: Valores, cantera y educación.

\section{Training in values from the Sports Psychology Area of Sevilla F.C. S.A.D.}

ABSTRACT: One of the main motivations of football club Sevilla F.C is to educate in values and to provide training to its players. This is considered as an essential objective of the work carried out by the Sports Psychology Area with the lower ranks of the club. Education in values seeks that players acquire behaviors, knowledge and attitudes appropriate to life and sports, such as respect, tolerance and fair play. The purpose of this article is to discuss some of the pillars and projects of the Sports Psychology Area of Sevilla FC in relation to education and integral formation of its players, emphasizing the schools in values for families, the management of players in the residence, the psycho-pedagogical support provided to academy players, and the school project "Take the school's game". It is concluded that counselling to parents, training in values, and formal education contribute to the personal growth and sports development of young players.

KEYWORDS: Personal values, academy and education.

\section{Formação em valores da Área de Psicologia do Desporto do Sevilha F.C. S.A.D.}

RESUMO: Uma das principais motivações do Sevilla F.C é educar em valores e treinar os seus jogadores, destacando-se como uma tarefa fundamental dentro do trabalho realizado pela Área de Psicologia do Desporto com os escalões inferiores do clube. A formação em valores procura que os jogadores adquiram comportamentos, conhecimentos e atitudes adequados à vida e ao desporto, como o respeito, a tolerância e o fair-play. Os objetivos do presente trabalho são expor alguns dos pilares e projetos da Área de Psicologia do Desporto do Sevilla FC em relação à educação e formação integral dos seus jogadores, destacando a formação em valores para as famílias, a gestão dos jogadores da residência, o apoio psicopedagógico à academia e ao projeto escolar "Joga à bola na escola". Conclui-

\footnotetext{
Juan Manuel Gamito Manzano, es psicólogo. Área de Psicología del Sevilla FC. SAD; Mercedes Valiente Martín, es psicóloga. Área de Psicología del Sevilla FC. SAD; José Alejandro Domínguez Catalán, es psicólogo. Área de Psicología del Sevilla FC. SAD; José Ignacio Navarro Díaz, es psicólogo. Área de Psicología del Sevilla FC. SAD; Alejandro Correa Palacios, es pedagogo. Área de Psicología del Sevilla FC. SAD.
} 
se que o aconselhamento aos pais, a formação em valores e os estudos contribuem para o crescimento pessoal e o desenvolvimento desportivo dos jovens jogadores.

PALAVRAS-CHAVE: Valores, academia e educação.

Artículo recibido: 10/02/2018 | Artículo aceptado: 08/05/2018

Desde el Área de Psicología de los escalafones inferiores del Sevilla F.C, se tiene muy en cuenta las consideraciones que Amat y Batalla (2000) establecen para que pueda producirse una educación en valores como base del éxito para el deporte y la vida de los deportistas (Álvarez, Cabo y García-Naveira, 2015). Es decir, practicarlo de modo que se permitan el fomento del autoconocimiento y la mejora del autoconcepto, la potenciación del diálogo como el mayor medio a la hora de resolver conflictos, la participación de todos, la potenciación de la autonomía personal, el aprovechamiento del fracaso como elemento educativo, la promoción del respeto y la aceptación de diferencias individuales y el aprovechamiento de las situaciones de juego para trabajar las habilidades sociales destinadas a facilitar la convivencia (Villalonga, Garcia-Mas, De las Heras, Buceta y Smith, 2015).

Siendo así, se asume que educar en valores es un proceso del día a día en el que sin duda tienen un papel influyente los padres y madres, entrenadores, profesores, así como su grupo de iguales (Leo, García-Calvo, Sánchez-Miguel, Gómez y Sánchez-Oliva, 2008; Leo, Sánchez-Miguel, Sánchez-Oliva, Gómez y García-Calvo, 2009).

Uno de los agentes activos en el trabajo, desarrollo y aplicación de valores, son los entrenadores, que en cada sesión de entrenamiento, partido, viaje, convivencia, entrenan y exponen una serie de valores que son útiles tanto en la vida deportiva como fuera de los terrenos de juego (Navarro y Martín, 2015). Algunos ejemplos son:

- Solidaridad / cooperación: etendida como un "proceso social a través del cual el rendimiento se evalúa y se recompensa en términos de los logros deportivos de un grupo de personas que trabaja en conjunto para alcanzar un objetivo particular" (Coakley, 1994).

- Compromiso: entendido como «una disposición psicológica que representa el deseo y la decisión de seguir participando en el deporte» (Scanlan, Simons, Carpenter, Schmidt, y Keeler, 1993). El clima motivacional en cada sesión de entrenamiento y las oportunidades para jugar y crecer son determinantes en el desarrollo de este valor por parte de los técnicos.

- Fair play: entendiéndolo no sólo como una forma de participar sino también como una manera de proyectarse en la vida con valores morales (Hon y O'connor, 1994). Los entrenadores inculcan en los jugadores la idea de la com- petición deportiva como una oportunidad para medir sus cualidades y potencial; a los equipos rivales no como enemigos sino como parte necesaria en el desarrollo de esas cualidades y la práctica del fútbol. Esta es una oportunidad de experimentación constante de actitudes de respeto hacia los demás, siempre presentes en la vida cotidiana.

Teniendo en cuenta, por último, que los/as niños/as adquieren y modifican patrones complejos de comportamientos, conocimientos y actitudes a través de la observación de los adultos y otros modelos cercanos -figuras de autoridad y personas a las que admiran-, el modelado es uno de los baluartes de nuestras actividades (Riviére, 1992).

A continuación se detalla algunas de las intervenciones en las que, bien directa o indirectamente, se trabajó la formación integral en la que se contemplan los valores deportivos y no deportivos como pilares fundamentales (Gómez-Espejo, Aroca, Robles-Palazón y Olmedilla, 2017). En primer lugar, enfocando en el rol de las familias en esta misión. En segunda instancia, se describe la perspectiva de la formación en valores desde la residencia de jugadores bajo la disciplina del Sevilla F.C. En tercer lugar, la integración de esta formación en el proyecto de apoyo psicopedagógico que, con éxito, se desarrolla con menores de los escalafones inferiores en situaciones escolares de riesgo y/o dificultad. Y, por último, el programa estrella de la Fundación del Club, denominado "Sácale Partido al Cole", que visita los colegios de Sevilla y alrededores con los jugadores del Sevilla F.C, organizando diferentes actividades para transmitir valores como la tolerancia, el respeto al rival o la importancia de una buena alimentación.

\section{Influencia de la familia en la formación en valores deportivos}

Una de las tareas fundamentales dentro de la práctica y enseñanza deportiva en general, es la de educar y formar en valores, tanto humanos como deportivos, desde temprana edad (Danioni, Barni y Rosnati, 2017). La filosofía de trabajo desde los escalafones inferiores de esta entidad deportiva parte de una base fundamental: la formación integral del deportista. Es decir, formar al deportista no solo a nivel deportivo, sino también a través de una formación humana, 
compuesta por una enseñanza y orientación académica y profesional, una educación en valores deportivos y un entrenamiento en habilidades sociales (Álvarez et al., 2015). Con esto, se quiere señalar la importancia de formar al jugador/a desde que se comienza a entrenar.

Para lograr la formación humana y deportiva que se busca, es crucial la formación en valores deportivos que se transmitan desde la familia (Ortiz, 2017). De esta manera, tomando como ejemplo el modelo de expectativa propuesto por Eccles y Harold (1991, citado en Esnaola y Revuelta, 2009), nos centramos en la importancia del entorno familiar a modo influencia (positiva o negativa) que tiene a lo largo de la práctica deportiva, relacionándose con el autoconcepto y la percepción de dificultad de la tarea que va a ir desarrollando el menor. Vemos, por tanto, como el entorno que envuelve el ambiente deportivo donde se forman los jugadores es un medio de influencias socializadoras, las cuales todas ellas van a sumar un papel relevante en la formación de las características del niño/a desde temprana edad (Boixadós, Cruz, Torregosa y Valiente, 2004, citado en Cruz et al., 2007).

Convirtiéndose en tarea fundamental contar con el apoyo de la familia para transmitir valores como la diversión, el apoyo y cooperación, compromiso, responsabilidad, obediencia, cuidado para la salud, etc. Esto se logra a partir de un trabajo en profundidad y a largo plazo, que comienza desde que el jugador/a entra en la práctica deportiva de un club de élite. Como ejemplo de tareas que ayudan a transmitir estos valores deportivos a los padres y madres, a continuación, describiremos el proyecto formativo denominado como "escuela de valores deportivos del Sevilla F.C".

Dicho proyecto se desarrolla a través de la impartición de charlas y talleres prácticos, con carácter mensual, a los familiares de nuestros jugadores/as, las cuales tienen una duración aproximada de 90 minutos y son llevadas a cabo por uno de los psicólogos el club. El contenido de las mismas se divide entre un contenido teórico y una parte de resolución de casos prácticos. A continuación se presenta el tema principal de algunas de las sesiones impartidas durante la temporada 2017/18, durante los meses de octubre a febrero, a los familiares de jugadores/as de la etapa de Fútbol 7 (benjamines y alevines):

- Importancia de la formación académica.

- Educación en valores deportivos para "padres entrenadores": diversión vs competición.

- Importancia de una alimentación sana y equilibrada.

- Uso de las redes sociales y nuevas tecnologías.

- Gestión y resolución de conflictos familiares: cooperación en el hogar.

El objetivo final de estas actividades es la de, tal y como describe Vila (1998, p.502, citado en Capano y Ubach, 2013), "proveer modelos adecuados de prácticas educativas en el contexto familiar y/o modificar y mejorar prácticas existentes con el objeto de promover comportamientos en los hijos e hijas que son juzgados positivamente y erradicar los que se consideren negativos". De esta manera, se hace congruente el trabajo realizado desde el área de psicología deportiva con el que realiza la familia desde su hogar, promoviendo entre todos estos valores deportivos positivos, ayudando por tanto a aportar aspectos relevantes para la formación integral del jugador/a.

\section{La residencia del Sevilla F.C como fábrica de valores}

Más de 15 años avalan a la "La casa del jugador sevillista", esta cuenta con todo lo necesario para una estancia acorde a las necesidades de los jóvenes jugadores que recalan en el club y tienen que estar separados de su entorno familiar (Morilla, Pérez, Gamito, Gómez, Sánchez y Valiente, 2003). El Sevilla F.C persigue la excelencia en todos los ámbitos de su desarrollo, para eso crea "una nueva familia"; en la actualidad, esa gran familia sevillista está formada por 45 jugadores y un grupo de profesionales (psicopedagogo, educadores y psicólogos) velando por el desarrollo integral del jugador, apostando por afianzar la excelencia de la cantera y garantizando el futuro para nutrir al primer equipo en la elite del fútbol internacional.

El valor de la cantera son los jugadores, lo cual se demuestra temporada tras temporada. ¿Cómo crear "un valor" con valores? Como se recoge en numerosos estudios (Cruz et al., 1996; Gimeno, 1995; Greendorfer, 1992) uno de los principales responsables sobre el desarrollo psicológico y físico en la práctica deportiva de los jóvenes deportistas son los padres; teniendo en cuenta esa cuestión, nos encontramos con una "institucionalización" del jugador-residente ocupando el equipo educativo en gran medida un "rol adjunto" a las figuras parentales.

El apartarse de su entorno familiar hace que se vean alteradas y queden al descubierto esas necesidades, las cuales son asumidas por los educadores y personal de residencia convirtiéndose en agentes socializadores de suma importancia para nuestros jugadores. Suplir no sustituir, compensar la carencia de sus figuras afectivas y sociales de referencia diariamente, a través de la "convivencia, constancia, trabajo y disciplina que son los pilares básicos que hacen realidad a futbolistas y personas competentes, modelos para los jóvenes, ejemplos para sus continuadores, y orgullo para todos los sevillistas (Morilla y Pérez, 2002). En la dinámica de trabajo de residencia nos enmarcamos en la siguiente máxima: "Crecer, aprender, respetar y competir". 
Crecer desde la responsabilidad, la pertenencia al grupo y su identificación al Club. Lo prioritario y fundamental para la integración en la dinámica del funcionamiento para un nuevo jugador que llega a nuestras instalaciones es "sentirse como en casa". La responsabilidad se gestiona desde la autonomía en sus acciones marcadas por un reglamento de orden y funcionamiento, buscando la cohesión del grupo de residentes (Chicau, Silva y Palmi 2012).

Aprender desde la humildad y el esfuerzo. Cuando recala un nuevo jugador en el Club se pone en valor la experiencia de los demás compañeros que llevan más tiempo en la entidad. La utilización de modelos competentes adecuados de referencia es útil para una adaptación y aprendizaje de comportamiento dentro de la dinámica de relación del grupo de jugadores (Buceta, 2004). Establecimientos de objetivos en los diferentes aspectos del aprendizaje del jugador: deportivo, académico y humano, desarrollando un trabajo integral basado en entrenamientos en habilidades sociales, resolución de problemas y reestructuración cognitiva con un estilo asertivo. Referente de ello es Pablo Blanco, Director General de Cantera, $2^{\circ}$ jugador canterano con el mayor número de partidos vistiendo la camiseta del Sevilla F.C en su historia y más de 20 años en Dirección de Cantera. Muestra de constancia, esfuerzo, preparación e identidad con el Sevilla F.C.

Respetar desde la norma y la tolerancia. Las normas de convivencia en la Residencia son extrapoladas a las situaciones deportivas, existiendo una línea de intervención coordinada entre la parte deportiva y el trabajo de los educadores de residencia. La casa del jugador sevillista se ha convertido en un ejemplo de tolerancia e integración, reflejo de una entidad abierta al respeto de creencias, culturas, nacionalidades y razas. Su integración nos permite trabajar desde la riqueza y la aportación de la diversidad a nuestras vidas, igualmente que el aumentar el repertorio técnico nos hace mejores jugadores, también el conocer y aprender de los demás nos hace ser más tolerantes, con mayores recursos y habilidades sociales, favoreciendo las integración en diferentes grupos y equipos que se formarán a lo largo de la vida deportiva de los jugadores.

Competir desde lo cotidiano a lo extraordinario, desde los entrenamientos a la competición (Chamorro, Torregrosa, Sánchez y Amado, 2016). El esfuerzo cotidiano es la seña de identidad de nuestro trabajo como club, desde la humildad del día a día; esforzarse se entiende para los sevillista como "nunca se rinde", un esfuerzo en exprimir las posibilidades personales y deportivas hasta sus últimas consecuencias, hasta el último segundo del partido como lo ha demostrado nuestra historia como club, marcando un ritmo de crecimiento sin olvidar la excelencia dentro de nuestro deporte consiguiéndolo desde la constancia, la humildad y la cotidianidad de poseer buenos hábitos saludables, cooperar, respetar a los demás y sentirse parte de una entidad como el Sevilla F.C.

El principal trabajo en valores se lleva a cabo en el día a día con la convivencia del grupo de educadores y los jugadores residentes (personal educativo permanece durante las 24 horas del día en la residencia). Se realizan intervenciones individuales y grupales de duración aproximada de 45 minutos, con carácter semanal, complementadas con intervenciones telefónicas con los familiares de los jugadores residentes. Las sesiones son realizadas por un psicólogo del club, los contenidos sobre los que habitualmente se trabajan son los siguientes:

- Importancia de la formación académica.

- Gestión y resolución de conflictos en la convivencia.

- Importancia de una alimentación sana y equilibrada.

- Respeto a nuestro entorno: organización y cuidado de nuestros espacios. (habitaciones, salas comunes, sala de estudio, comedor, furgoneta, autobús,...)

- Aumentar nuestra responsabilidad y asumir diferentes roles deportivos durante la temporada (lesión, titularidad, suplencia).

\section{Trabajar valores desde el proyecto psicoeducativo}

En el proyecto psicoeducativo se trabaja en la misma línea de transmisión de valores bajo la filosofía de cantera e identidad del club que se puede trabajar en el resto de áreas. Estas bases educativas y formativas de la entidad se ven reforzadas por Arnold (1991), el cual nos recuerda la creencia tradicional de que a través de la participación en juegos y deportes surgen cualidades como lealtad, cooperación, valor, resolución, fuerza de voluntad, dominio de sí mismo, resistencia, perseverancia o determinación.

En este proyecto los valores se llevan a cabo a través del ámbito académico, con el objetivo de conseguir que jugador/a y alumno/a sean la misma persona, y que éste/a vaya en consonancia en las diferentes parcelas de su vida. El extrapolar los valores transmitidos a través del deporte a los estudios y viceversa garantizará una interiorización de los mismos donde le será más fácil de aplicar y llevarlos a la práctica, alcanzando así el éxito en la formación integral de la persona (Gutiérrez, 1995).

Con la aceptación del jugador a entrar en el proyecto se trabaja el compromiso; los/as jugadores/as asisten todos los días de entrenamiento una hora a clase antes del comienzo de la sesión, en esta área se les exige la misma puntualidad y seriedad que en las diferentes áreas de preparación, al igual que con los diferentes entrenadores, técnicos y profesionales. Igualmente se trabaja dicho compromiso con la 
asistencia regular y no faltar bajo causa justificada, así se refuerza además el hábito y rutina de estudio.

El esfuerzo se evidencia en la necesidad de tener que llegar a la ciudad deportiva una hora antes de lo que lo haría en circunstancias normales, renunciando a tiempo libre para invertir en la mejora académica.

Todo continúa con la constancia y perseverancia, primordiales para asimilar los nuevos hábitos y llevarlos a cabo en el día a día, dejándose ver estos valores en la asistencia diaria y en la realización de la tarea escolar; constancia y perseverancia porque los buenos resultados de estas nuevas conductas se obtendrán a medio-largo plazo y se verán reflejados en las notas de los exámenes y/o en las calificaciones trimestrales.

Aun así, los jugadores y jugadoras deben aprender el valor del sacrificio, ya que, sus horarios y desgaste físico, psicológico y emocional no es el mismo que el de sus iguales, se les inculca a rendir en momentos y situaciones adversas, así, desde este proyecto, se les enseña a administrar y gestionar los tiempos.

Recordando que los/as protagonistas de este proyecto son menores que destacan en el fútbol pero necesitan mejorar en los estudios, el valor de afán de superación y motivación es inversamente proporcional del área deportiva respecto al área académica; por eso, en este proyecto es muy importante mostrarles y reforzarles los aprendizajes y mejoras por mínimas que sean. Para alcanzar este valor, sin dejar la línea educativa, formativa y competitiva, hacemos a que "la sana competitividad es la que nos lleva a esforzarnos, a dar lo mejor de nosotros mismos, a progresar, a buscar nuevas estrategias para superarnos, a descubrir y aprender de nuestros errores y a buscar nuevas soluciones para mejorar. Sin ella no progresaríamos".

La autoconfianza y autoestima se trabaja de manera transversal en este proyecto mediante el valor de la resolución de adversidades, por ejemplo, ayudándoles a que vean que dedicándole tiempo a un ejercicio pueden terminar realizándolo bien y encontrar la solución, y aunque en un principio creyeran no saber hacerlo, parándose a leer y comprender la teoría encontrarán la respuesta al problema ellos solos.

Mediante el uso correcto de la agenda y los materiales necesarios (cuadernos, libros, estuche...) se fomenta la responsabilidad.

Tratándose de un proyecto donde coinciden en la misma aula jugadores y jugadoras de diferentes categorías, diferentes equipos, cursos y edades, el valor del respeto es fundamental; respeto a los/as compañeros/as, así como respeto al momento de estudio en silencio, respeto a la concentración que se necesita para realizar correctamente unas actividades, respeto a la atención que requiere una explicación.
Cabe destacar, que los valores desarrollados anteriormente son los que se trabajan de una manera más explícita en el proyecto, sin embargo, existe una gran lista de más valores que se trabajan y se llevan a cabo de un modo subliminal en el día a día del equipo profesional que trabaja con estos/ as futuros futbolistas, puesto que, lo importante a la hora de inculcar valores es el propio comportamiento que muestre el educador, profesor o entrenador durante la actividad, mucho más importante que los discursos teóricos.

\section{Más allá del Club: programa "sácale partido al cole"}

Es una realidad el auge, cada vez más notorio, que los clubes de fútbol tienen en el mundo de los jóvenes y también lo que supone para ellos su equipo y los jugadores que lo representan, siendo éstos verdaderos referentes para sus vidas.

Por todo ello, las entidades deportivas no deben estar ajenas a esa circunstancia, bien al contrario, deberían aportar todo aquello que esté a su alcance y que redunde en una mejora en la formación en todos aquellos aspectos que deben adornar a un buen ciudadano que se precie, sea éste joven o adulto. Reconociendo todo lo anteriormente expresado, sólo queda desde los distintos clubes deportivos su implicación en esa educación integral de futuros ciudadanos, como por ejemplo en los estudios de sus jugadores (García-Naveira y Jerez, 2012).

El deporte es un lugar privilegiado para realizar una educación humanizadora. De esta forma, se podrá favorecer el crecimiento y maduración integral de la persona, aportando valores que formarán su personalidad (Corrales 2010). De aquí surgen valores como el compromiso y el respeto, constructos fundamentales para construir la "deportividad" (Peñaloza, Andrade, Jaenes y Méndez, 2013).

El Sevilla F.C, con más de cien años de historia, aprovechó las numerosas actividades de su centenario para mirar al ámbito educativo, a esos jóvenes aficionados o no al deporte para ofrecerles una serie de actividades que contribuyesen a una correcta formación en valores.

Es así como el Sevilla F.C se acerca a la escuela pública, privada y concertada, reconociendo la supremacía de ésta sobre cualquier otra institución, pero entendiendo también la importante ayuda y colaboración que puede prestar a la educación integral de los jóvenes.

Pensamos dar a conocer a nuestros escolares el fútbol de una forma "no convencional", mediante actividades abiertas en las que la diversión, la participación, la cooperación y la no competitividad, contribuyan a que organicen mejor su segundo y tercer tiempo pedagógico. 


\section{Objetivos del programa}

a. Contribuir a la educación de los jóvenes ya que la Educación es tarea de todos. El Sevilla F.C aporta su granito de arena a esa sociedad formando jóvenes en los valores deportivos en lo que se denomina "intromisión colaboradora".

b. Exportar a las aulas el poder socializador y educativo del fútbol y los beneficios que puede reportar a nuestros jóvenes, trasladando los valores de la tolerancia y la solidaridad entre personas.

c. Dar una visión más humana y cercana del deporte, donde lo que tenga valor sea la persona, sus intereses y sus necesidades y donde todo el resto quede subordinado a esta premisa.

d. Transmitir a los escolares valores como, Tolerancia, Respeto, Estilo de vida saludable, Integración racial y cultural, como parte de nuestra campaña "cordiality"

\section{Desarrollo del programa escolar}

Día 1. Actividades en el centro escolar: la primera actividad es la de presentar a los alumnos las actividades que se van a llevar a cabo en su centro y entrega de camisetas del programa escolar.

1. Concurso de redacción y dibujo. Se realiza a lo largo de la semana siguiente un primer y segundo ciclo de primaria y un concurso de redacción para el tercer ciclo, que está relacionado con la salud, educación y deporte.

2. Entrega y explicación del material formativo sobre valores: a. Libro para alumnos/as: cuadernillos de contenidos formativos.

b. Libro para padres: cuadernillos con contenidos formativos.

c. Unidades didácticas: de iniciación al fútbol para profesores de Educación Física.

3. Actividad deportiva (postas de ejercicios habituales en un entrenamiento de jugadores/as de fútbol en edad escolar de nuestra cantera, promoviendo la participación mixta).

4. Charla alimentación sana y salud bucodental, dirigida por especialistas en la materia. El objetivo de esta actividad es promover los hábitos sanos en la alimentación para lo que se imparte una charla sobre nutrición, de forma dinámica y lúdica. Esta charla es enlazada con otra sobre cómo prevenir gran cantidad de enfermedades con una buena higiene de la boca a los alumnos-as del primer ciclo de primaria. Todo esto se lleva a cabo en forma de cuentacuentos.

5. La cámara del pensamiento. Proponemos esta actividad donde los alumnos-as en número de 2-3 se enfrentan a una cámara a la que deben exponer sus pensamientos sobre varios temas propuestos: deportividad, amistad, tolerancia, cooperación, esfuerzo, fútbol...

Día 2. Visita del centro al Estadio Sánchez Pizjuán. Esta visita la realizan un máximo de 100 alumnos que acuden al estadio desde su centro en autobuses, a los 10-15 días de la visita al centro, seguido el acontecimiento por los medios oficiales del club. :

- Entrada al estadio y entrega de camiseta conmemorativa para cada alumno-a y profesorado.

- Desayuno saludable, consistente en zumo y pan con aceite de oliva.

- Charla Formativa sobre Valores por parte de técnicos de la cantera.

- Tour por el Estadio.

- Sala 1890. Un experto del área de historia explica nuestros orígenes apoyado en un vídeo ilustrativo.

- Antepalco. Aquí pueden ver la Copa de la UEFA Europa League 15-16 así como el resto de copas en nuestras vitrinas.

- Vestuario. Los alumnos-as visitan una de las zonas más privadas del estadio, sintiéndose como jugadores profesionales de fútbol por un día.

- Terreno de juego. Se visita el propio terreno de juego y banquillos.

- Charla cordiality: El objetivo de esta actividad es educar a los niños /as como buenos aficionados/as al deporte, teniendo muy en cuenta la tolerancia y el respeto hacia las otras aficiones, tanto en los estadios como fuera de ellos, apoyados sobre un vídeo de buenas prácticas.

- "Rueda de prensa escolar" (emitida en directo por SFC TV).

- Mensaje del Presidente del Sevilla F.C y dos jugadores de la primera plantilla sobre aspectos educativos y de salud.

- Preguntas a presidente y jugadores sobre cordiality y otros temas.

- Entrega de Premios: Entrega por parte del presidente junto a los jugadores de los premios a los mejores trabajos realizados de redacción y dibujo.

- Firmas y Regalos a los alumnos/as: firma de presidente y jugadores, así como diferentes regalos.

- Salto al terreno de juego: veintidós alumnos/as preseleccionados por el propio centro se visten con equipaciones oficiales del Sevilla F.C y la escuela deportiva Antonio Puerta. A modo de inicio de partido oficial de la Liga de Fútbol Profesional, saliendo cada equipo acompañado de un jugador, y junto al presidente, saltando al terreno de juego.

- La despedida de los escolares concluye con la entrega de un picnic a cada niño/a.

Día 3. Invitación a un partido en el Ramón Sánchez Pizjuán. Los participantes del programa escolar son invitados a un partido oficial del Sevilla FC para que vivan en directo un partido como aficionados/as en nuestro Estadio y pongan en práctica las buenas costumbres que se les han transmitido. 
Día 4. Gran fiesta escolar. Como cierre del Programa Escolar de cada curso, se lleva a cabo una gran Fiesta Escolar en el Parque Temático Isla Mágica, en la que se elige al ganador final anual del Programa, haciéndole entrega de un abono para la siguiente temporada, con la presencia del Presidente, así como los jugadores de la Primera Plantilla.

Paralelamente, distribuidas estratégicamente por el Parque Temático, hay mesas en las que se llevan a cabo sesiones de autógrafos por parte de jugadores.

En definitiva, una amalgama de actividades tanto internas como externas a los propios dominios del Club con las que se intenta aportar en la formación en valores de las generaciones venideras.

\section{Discusión}

El Sevilla F.C pone un enorme interés en todo lo referente a transmitir una educación en valores, desde la entidad se marca siempre un nivel de excelencia, esto hace que este aspecto se trabaje con la misma profesionalidad e importancia que otras cuestiones dentro del club. La intervenciones centradas en los Escalafones Inferiores se debe al pensamiento de ser un club que apuesta por crecer y formar a sus jugadores desde las edades más tempranas, siendo un pensamiento de solidez e identidad en los valores que han hecho grande a este club. Además la grandeza de este Club y sus éxitos deportivos sobre todo europeos nos ha llevado a estar inmerso en estos últimos años en programas internacionales como el Proyecto Europeo PsyTool o Proyecto Internacional ELIT-in.

Como quedó recogido, el Sevilla F.C realiza una apuesta firme sobre el trabajo en valores y los profesionales que lo llevan a cabo. Siendo desde hace años el club de fútbol de primera división que tiene en su plantilla a más psicólogos para poder desarrollar un trabajo adecuado, supervisado y sujeto a la profesionalidad que se requiere en un club del nivel del Sevilla F.C. Se considera de gran relevancia la aportación de los profesionales de la psicología del deporte dentro de cada uno de los proyectos antes reseñados, así como en cada uno de los equipos de los escalafones inferiores. La mejora del rendimiento deportivo se sustenta en un trabajo interprofesional y en el Sevilla F.C no se entiende sin la aportación del Área de Psicología. Esto es otra muestra más de la integración y convivencia que desde los profesionales se transmite a nuestros jugadores/as y a las familias de estos.

Sabemos desde el Área de Psicología del club, que siempre tenemos la obligación y el compromiso profesional de estar al nivel élite que destila la entidad, pero no es más cier- to que en ocasiones nos encontramos con algunas limitaciones para afrontar el volumen de intervenciones individuales, atenciones grupales de equipos, preparación de encuentros, supervisión y desarrollo nuevos proyectos. Gracias a una excelente coordinación e implicación en el trabajo cooperativo del área seguimos haciendo un trabajo consolidado y respetado por todos los profesionales del club.

El Sevilla F.C siempre aspira a cotas más altas cada temporada, en esa línea nos marcamos una fuerte revisión del Área de Psicología cada final de temporada. Con el fin de eliminar las complicaciones encontradas por el volumen de demanda que genera una entidad con más de 400 jugadores/as, proponiendo ajustes en la disposiciones de sesiones grupales que ayuden a reducir las intervenciones individuales. Es decir trabajar más la prevención, formar a "agentes en valores" y la implantación de un "currículo de valores individual y de equipo".

Por último, una de las apuestas más importantes del club es la mejora de las instalaciones de la Ciudad Deportiva José Ramón Cisneros Palacios, en este proyecto de ampliación y modernización está contemplado un nuevo programa para la implementación para educar en valores, desarrollado conjuntamente entre el Área de Psicología y el Departamento de Marketing en el diseño y señalética de paneles con mensajes adaptados a transmitir los valores del club y del deporte.

\section{Referencias}

Álvarez, M. A., Cabo, J. A. y García-Naveira, A. (2015). Aprendizaje y formación para el éxito deportivo y personal. En A. García-Naveira y L. Locatelli (Eds.), Avances en Psicología del Deporte (pp. 335-354). Barcelona, España: Paidotribo.

Amat, M. y Batalla, A. (2000). Deporte y educación en valores. Aula de Innovación Educativa, 91, 10-13.

Arnold, P. J. (1991). Educación Física, movimiento y currículum. Madrid, España: Morata.

Boixadós, M., Cruz, J., Torregrosa, M. y Valiente, L. (2004). Relationship among motivational climate, satisfaction, perceived ability and fair play attitudes in young soccer players. Journal of Applied Sport Psychology, 16, 301-317. https://doi. org/10.1080/10413200490517977

Buceta, J. M. (2004). Estrategias psicológicas para entrenadores de deportistas jóvenes. Madrid, España: Dykinson.

Capano, A. y Ubach, A. (2013). Estilos parentales, parentalidad positiva y formación de padres. Ciencias Psicológicas, 7(1), 83-95.

Chamorro J. L., Torregrosa M., Sánchez O. D. y Amado, D. (2016). El fútbol dentro del campo y fuera de él: desafíos en la transición a la élite. Revista de Psicología del Deporte. 25, 81-89.

Chicau, C., Silva, C. y Palmi, J. (2012). Programa de Intervención psicológica para la optimización del concepto de equipo (team building) en jóvenes futbolistas. Revista de Psicología del Deporte, 21, 49-58. 
Coakley, J. (1994). Sport in society: Issues and controversies. St. Louis: Times Mirror/Mosby.

Corrales, A.R. (2010): El deporte como elemento educativo indispensable en el área de educación física. Revista Digital de Educación Física, 1(4), 23-36.

Cruz, J., Capdevila, LI., Boixadós, M., Pintanel, M., Alonso, C., Mimbrero, J. y Torregrosa, M. (1996). Identificación de conductas, actitudes y valores relacionados con el fairplay en deportistas jóvenes. En Cruz, J. (Ed.), Valores sociales y deporte. Fair play versus violencia. Investigaciones en ciencias del deporte (pp. 3767). Madrid: Consejo Superior de Deportes.

Cruz, J., García-Mas, A., Palou, P., Sousa, C., Torregrosa, M., Viladrich, C. y Villamarín, F. (2007). La influencia de padres y madres en el compromiso deportivo de futbolistas jóvenes. Revista Latinoamericana de Psicología, 39(2), 227-237.

Danioni, F., Barni, D. y Rosnati, R. (2017). Transmitting sport values: the importance of parental involvement in children's sport activity. Eur J Psychol, 13(1), 75-92. https://doi.org/10.5964/ejop. v13i1.1265

Eccles, J. S., y Harold, R. D. (1991). Gender differences in sport involvement: Applying the eccles' expectancy-value model. Journal of Applied Sport Psychology, 3(1), 7-35. https://doi. org/10.1080/10413209108406432

Esnaola, I. y Revuelta, L. (2009). Relaciones entre la actividad física, autoconcepto físico, expectativas, valor percibido y dificultad percibida. Acción Psicológica, 6(2), 31-43. https://doi. org/10.5944/ap.6.2.219

García-Naveira, A. y Jerez, P. (2012). Departamento de psicología del club Atlético de Madrid: filosofía, programación y desempeño profesional en el fútbol base. Cuadernos de Psicología del Deporte, 12(1), 111-120. https://doi.org/10.4321/S157884232012000100010

Gimeno, F. (1995): La adecuación del desempeño de los roles paterno y materno como variable facilitadora de la práctica del deporte de competición en el joven deportista. Boletín de la Sociedad de Pediatría de Aragón, La Rioja y Soria, 25(2), 58-61.

Gómez-Espejo, V., Aroca, B., Robles-Palazón, F. y Olmedilla, A. (2017). Formación integral en la cantera del Real Murcia C.F.: tutorías psicológicas. Revista de Psicología Aplicada al Deporte y al Ejercicio Físico, 2(e11), 1-11. https://doi.org/10.5093/rpadef2017a11

Greendorfer, S. L. (1992). Sport Socialization. En T.S. Horn (Ed.), Advances in sport Psychology (pp. 201-218). Champaign, Illinois: Human Kinetics.
Gutiérrez, M. (1995). Los valores sociales y Deporte. Madrid, España: Gymnos.

Hon, J. y O'connor, B. (1994). Teaching Fair Play: The Essence of Sport. Journal of Physical Education recreation and dance. 67(7). 70-72. https://doi.org/10.1080/07303084.1994.10606969

Leo, F. M., García-Calvo, T., Sánchez-Miguel, P. A., Gómez, F. R., y Sánchez-Oliva, D. (2008). Relevancia de los climas motivacionales de los otros significativos sobre los comportamientos deportivos en jóvenes deportistas. Motricidad. European Journal of Human Movement, 21, 123-141.

Leo, F. M., Sánchez-Miguel, P. A., Sánchez-Oliva, D., Gómez, F. R., y García-Calvo, T. (2009). Análisis de las relaciones existentes entre la orientación y el clima motivacional con los comportamientos antisociales en jóvenes deportistas. Revista Iberoamericana de Psicología del Ejercicio y el Deporte, 1, 15-28.

Morilla, M y Pérez, E. (Coords.) (2002). Entrenamiento de la Atención y Concentración. Ejercicios para su mejora. Sevilla: Difusión Gráfica.

Morilla, M., Pérez, E., Gamito, J., Gómez, M., Sánchez, J y Valiente, M. (2003). Planificación psicológica de la cantera del Sevilla F.C.S.A.D.: Organización, funcionamiento y programa deportivo-formativo. Cuadernos de Psicología del Deporte, 3(2), 17-30.

Navarro, J.I., y Martín, A, (2015). Atontados por el fútbol. Tipología de padres y entrenadores. Sevilla, España: Editorial Samarcanda.

Ortiz, P. (2017). No todo es Balón. Implicando positivamente a los padres en la práctica del fútbol en etapa formativa. Revista de Psicología Aplicada al Deporte y al Ejercicio Físico, 2(e9), 1-10. https://doi.org/10.5093/rpadef2017a9

Peñaloza, R., Andrade, P., Jaenes, J. C. y Méndez, P. (2013). Compromiso deportivo en jóvenes mexicanos. Revista Iberoamericana de Psicología del Ejercicio y el Deporte, 8(2), 317-330.

Riviére, A. (1992). Teoría Social del Aprendizaje: Implicaciones Educativas. En Coll, C., Palacios, J. y Marchesi, A. (Comp.), Desarrollo Psicológico y Educación I/ (pp-69-80). Madrid: Alianza.

Scanlan, T.K., Simons, J.P., Carpenter, P.J., Schmidt, G.W., y Keeler, B. (1993). The sport commitment model: Measurement development for the youth-sport domain. Journal of Sport and Exercise Psychology, 15, 16-38. https://doi.org/10.1123/jsep.15.1.16

Vila, I. (1998). Familia, escuela y comunidad. Barcelona: I.C.E. Universidad de Barcelona. Horsori

Villalonga, T., Garcia-Mas, A., De las Heras, R., Buceta, C. y Smith, R. (2015). Instauración y tareas de un servicio de psicología del deporte en un club de fútbol profesional. Revista de Psicología del Deporte, 24(2), 319-326. 